\title{
LRcell: detecting the source of differential expression at the sub-cell type level from
}

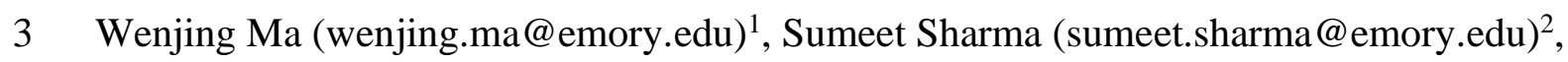

4 Peng Jin (peng.jin@emory.edu) ${ }^{3}$, Shannon L. Gourley (shannon.l.gourley@emory.edu) ${ }^{4}$,

5 Zhaohui Qin (zhaohui.qin@emory.edu) ${ }^{1,5, *}$

6

7 * Correspondence: zhaohui.qin@emory.edu

$8{ }^{1}$ Department of Computer Science, Emory University, 400 Dowman Drive, Atlanta, GA

930322, USA

$10{ }^{2}$ Graduate Program in Neuroscience, Emory University, 1462 Clifton Road NE, Atlanta, GA

1130322, USA

$12{ }^{3}$ Department of Human Genetics, Emory University, 1365 Clifton Road, Atlanta, GA 30322,

13 USA

$14{ }^{4}$ Department of Pediatrics, School of Medicine, Emory University, 100 Woodruff Circle, 15 Atlanta, GA 30322, USA; Yerkes National Primate Research Center, Atlanta, GA 30322, 16 USA

$17{ }^{5}$ Department of Biostatistics and Bioinformatics, Rollins School of Public Health, Emory 18 University, 1518 Clifton Road NE, Atlanta, GA 30322, USA 


\section{Abstract}

27 Given most tissues are consist of abundant and diverse sub cell-types, an important yet

28 unaddressed problem in bulk RNA-seq analysis is to identify at which sub cell-type(s) the

29 differential expression occur. Single-cell RNA-sequencing (scRNA-seq) technologies can

30 answer the question, but they are often labor-intensive and cost-prohibitive. Here, we present

31 LRcell, a computational method aiming to identify specific sub-cell type(s) that drives the changes observed in a bulk RNA-seq experiment. In addition, LRcell provides pre-embedded marker genes computed from putative single-cell RNA-seq experiments as options to execute

34 the analyses. Using three different real datasets, we show that LRcell successfully identifies

35 known cell types involved in psychiatric disorders and LRcell is more sensitive than even the 36 leading deconvolution methods.

37 Keywords: Cell-type enrichment, Cell marker genes, Differential gene expression 


\section{Background}

53 Finding genes that are differentially expressed (DE) between experimental conditions is a

54 powerful approach to understand the molecular basis of phenotypic variation. However, most tissues are consisted of tens or even hundreds of diverse sub-cell types and DE may only occur in a small subset of these sub-cell types, which are relevant to the experimental condition. Bulk RNA-seq data alone is unable to reveal the sub-cell types that drives the DE.

The rapid development and proliferation of single cell technologies resulted in massive accumulation of single cell transcriptomics data (scRNA-seq) from diverse tissue types.

61 These data reveal substantial variations in transcriptional regulation among different cell types and offer an unprecedented close-up view of the modifications underlying important biological processes including which cell types drive DE [1]. However, steep cost and complicated protocols prevent the wide-spread adoption of scRNA-seq.

65

In this study, we propose a novel computational tool named LRcell. Given the result from a bulk RNA-seq DE study, the goal of LRcell is to delineate which sub-cell type(s) of the tissue underwent substantial changes between the two experimental conditions. Exploiting cell type-specific marker genes identified from generic scRNA-seq available from publicly available data repositories, LRcell achieves the goal by surveying the enrichment of marker genes across all sub-cell types in the tissue (Figure 1). Thus, no scRNA-seq experiment matching the bulk RNA-seq experimental condition is needed. When applying LRcell to a diverse panel of bulk RNA-seq DE experiments, we successfully identify known cell types involved in the pathogenesis of psychiatric disorders as well as produce testable new hypotheses that have the potential to produce fresh new biological insights. 


\section{Results and Discussion}

In this work, we collect and curate a compendium of marker genes from multiple published scRNA-seq datasets. We then conduct LRcell analysis on multiple bulk RNA-seq DE experiments to demonstrate its utility.

\subsection{Marker gene collection and sources}

Similar to a collection of gene set for Gene Set Enrichment Analysis (GSEA) [2], LRcell requires a compendium of high-quality cell type marker genes. Currently, LRcell package provides users with multiple pre-loaded marker gene sets from human blood, human brain and mouse brain (Figure 2.A), computed from scRNA-seq datasets. Additionally, LRcell package offers external cell markers collected by Molecular Signatures Database (MSigDB)

[3]. The external makers all originate from human species including midbrain, cord blood, ovary and skeletal muscle. We store all cell-type specific marker gene sets into another R Bioconductor ExperimentHub package named LRcellTypeMarkers. Additional marker gene sets are being tested and will be added to the collection.

\subsection{Microglia highly enriched in Neurodegenerative Dementia}

In a recent neurodegenerative dementia study, Swarup and colleagues contrasted TPR50 mice expressing tau mutant with wild type mice using bulk RNA-seq in order to identify gene networks mediating dementia [4]. To identify the cell type(s) most involved in the condition, we apply LRcell to the DE gene list using pre-embedded marker genes from adult mouse Frontal Cortex (FC) region [5]. From LRcell result, we observed that Microglia show up as highly significant (Figure 2.B) which is concordant with previous studies [6]. Additionally,

100 the FC_11-3.unknown and FC_11-4.unknown sub cell types also show high level of 
significance. No annotation is available for these two cell clusters in the original publication. unknown cell clusters have considerable overlaps with the FC_11-1, which is also a Microglia cell type (Figure S1. A), which explains the pattern we observe.

\subsection{CD16+ Monocytes highly enriched in Post-Traumatic Stress Disorder (PTSD)}

In a recent study, Breen and colleagues conducted a bulk whole-transcriptome study using peripheral blood leukocytes collected from U.S. Marines, among which some developed PTSD post-deployment [7]. Using this dataset, we generate a list of DE genes that show

110 significant difference between the PTSD group and the control group at the pre-deployment 111 timepoint.

113 Using human marker genes derived from a single cell transcriptomic study on PBMC [8],

114 LRcell analysis finds that cells annotated as CD16+ non-classical Monocytes shows up as the 115 most significant among all cell types in PBMC (Figure 2. E). Our finding makes biological sense because as stated in previous studies [9], heterogeneity exists in monocytes distinguished by CD16 surface proteins and non-classical monocytes have been validated to regulate immune responses in trauma $[10,11]$.

\subsection{Marker genes from different region or timepoints}

121 To apply LRcell, an important question is that which marker gene sets to use, i.e., how to select single cell RNA-seq data where the source of the tissue match the tissue type profiled in the bulk transcriptomic study. This is particularly important for complex tissues such as brain. To address this issue, we use the neurodegenerative dementia study [4] as an example, which contains information from four brain regions: cortex, hippocampus (HC), cerebellum 
126 (CB) and brain stem. Brain stem is excluded from our analysis due to the lack of marker gene

127 information from that region of the brain.

To understand how marker genes vary across brain regions, we first define marker genes in

130 all regions of the brain to explore their spatial pattern (Figure S1. B; Figure S1. C). We

131 observe that glia cells, such as Astrocytes, from different regions have higher number of overlapping marker genes which indicates the homogeneity of glia cells across the brain. In contrast, neurons and interneurons share very few marker genes across different brain

134 regions. We then apply pre-embedded adult mouse brain marker genes from FC, $\mathrm{HC}$ and $\mathrm{CB}$

135 to bulk DE genes obtained from cortex, $\mathrm{HC}$ and $\mathrm{CB}$ respectively (Figure S3). We observe that Microglia cells are highly enriched in all three brain regions whereas Astrocytes are particularly highly enriched in CB (Figure S3). Especially when applying CB marker genes to $\mathrm{CB}$ bulk DE experiment, we notice that one sub-cell type of Astrocytes is highly enriched compared to others. Our observations demonstrate that selected cell types are heterogeneous

140 spatially, meaning marker genes are highly specific not only for the cell type, but also which region the cell belongs to. Because of this finding, it is highly desirable to run LRcell using marker genes of cell types located in closely matched brain regions. conducted on non-normal samples is acceptable as the reference. To address this question, we 146 use data from the HIV vaccine study [8], we observe that the expression of cell-type specific marker genes is mostly consistent across different timepoints within the same cell type (such as CD8 cells), and distinct across different cell types (Figure S2.A; Figure S2.B). For example, B sub-cell types share a considerable number of marker genes across timepoints, 
are collected from control samples, we believe that marker genes identified from non-normal samples are acceptable when scRNA-seq data from normal samples are not available.

\subsection{Comparison to GSEA}

155 GSEA is a powerful tool to determine whether a pre-defined gene set show concordant shift

156 in expression when comparing two biological conditions. One could potentially replace

157 LRcell with GSEA to identify DE-driving cell types, by treating cell-type specific marker

158 genes as pre-defined gene sets. To compare performance of the two methods, we repeat the

159 analyses done in section 3.1 and 3.2 using GSEA. The GSEA result from the

160 neurodegenerative dementia mouse model (Figure 2C) yields several equally significant cell

161 types including Astrocyte, Endothelial, Microglia, Mural, Oligodendrocyte and

162 Polydendrocyte. The tied significances lead to difficulties in determining which cell type(s)

163 potentially participated in dementia pathogenesis. Similar pattern is observed in the GSEA

164 result on PTSD study (Figure 2F) which shows that Monocytes, Dendritic cells and some T

165 sub-cell types are equally enriched. Based on the above observations, we conclude that

166 LRcell is more effective than GSEA to identify sub cell types that are most impacted by the

167 condition change in bulk DE experiments.

\subsection{Comparison to MuSiC}

170 A plethora of deconvolution methods has been developed to infer the proportions of different

171 sub cell types from bulk transcriptomic data [1][12][13][14][15]. By deconvoluting

172 individual bulk samples and comparing their proportions, one can identify both cell-type

173 proportion and gene expression changes. Hence it is of interest to compare LRcell with such a

174 bulk deconvolution strategy. For comparison, we apply MuSiC [13], a deconvolution method

175 that have shown favorable performance, to the mouse neurodegenerative dementia study [4] 
on both conditions. Because some layers of neurons are predicted to have almost zero

177 proportion (Figure 2D) when using all 81 sub-cell types, we merge the original sub-clusters into 15 major cell types in order to achieve a better representation. Despite this, MuSiC does not detect significant difference in Microglia or Astrocyte in terms of their proportions

180 between the two conditions. When applied to the PTSD study, using the original cell cluster annotations, MuSiC shows that most of the T sub-cell types have zero proportion and the proportion of CD14+ Monocytes is up to $60 \%$ (Figure S2G). In contrast, LRcell produces more sensible results because it is not limited by the number of cell types as it can detect the subtle differences among sub-cell types.

\subsection{Discussions}

187 Detecting transcriptional activity changes at the individual cell type level, especially their 188 modifications in disease samples, is crucial for understanding the mechanisms of diseases 189 development. Deconvolution-based computational tools have been developed to dissect cell

190 type proportions from bulk gene expression profiles. The computationally inferred

191 proportions can then be used for comparison. However, when apply to real data, such a strategy often encounters numerical stability problem and unable to handle large number of sub-cell types. In this study, we propose a novel alternative strategy named LRcell, LRcell

194 conducts enrichment analysis of cell-type specific marker genes among the top (or bottom)

195 DE genes identified by bulk transcriptome studies. Cell types that show the most enrichment 196 is likely to play an important role in the condition alteration. When applying to real datasets, 197 we found LRcell can successfully identify the involvement of the Microglia and Astrocytes in 198 neurodegenerative dementia and rare Monocytes in PTSD. 
In spirit, LRcell operates similarly as GSEA. But LRcell is much more sensitive to minor differences in marker genes of sub-cell types, which indicates its potential to detect changes in sub-cell types caused by disease conditions. Additionally, when compared to existing bulk deconvolution methods, LRcell is more stable in its ability to handle the similarities among sub-cell types. Thus, LRcell enables researchers to glean new biological insights from the bulk transcriptomics experiments with no need of redo the experiment using single cell technology. We are currently applying LRcell to a diverse set of clinical studies (Sharma, personal communication) to generating more biological insights.

To enable straightforward comparison, currently, we select a fix number of 100 marker genes

210 from each sub-cell type. Understandably, the number of marker genes for different cell types

211 varies, it is desirable to allow flexibility in choosing the number of marker genes based on the

212 transcriptomic patterns across cell types. However, different numbers of marker genes post

213 challenge for conducting enrichment analyses fairly across all cell types. This will be

214 investigated in our future studies.

LRcell currently provides embedded marker genes from human blood, human brain and

217 mouse brain calculated from scRNA-seq experiments along with markers from 66 cell types

218 in four tissues (midbrain, cord blood, ovary, and skeletal muscle) adopted from MSigDB. We

219 are working to include more tissue types in the future releases of LRcell which will make it 220 more widely applicable.

\section{Conclusions}

223 In summary, we develop LRcell, an R Bioconductor package for identifying sub-cell type(s)

224 that drive the changes observed in bulk comparative transcriptomic studies, taking 
advantages of newly emerged scRNA-seq data. The rationale of LRcell is that we believe marker genes of the modifying cell types tend to be enriched toward the top (or bottom) of the DE gene lists. We conduct comprehensive surveys applying LRcell across various experimental conditions, and successfully identify cell types that play important roles in neurodegenerative dementia and PTSD. Hence, we believe LRcell can provide researchers important and new biological insights in terms of the source of the biological changes at the sub-cell type level, without the need of conducting costly and laborious scRNA-seq experiments.

\section{Methods}

\section{Basic Assumptions}

236 The goal of LRcell is to identify the most affected sub-cell type(s) during the transition of

237 experimental conditions using only bulk transcriptomic data. Based on the assumptions that

238 cell-type specific marker genes of key cell types tend to be over-represented among the

239 significant DE genes in bulk transcriptomic studies, LRcell can discover which cell type(s) is

240 involved in certain disease or condition change. In recent years, computational methods have

241 been developed to deconvolve bulk RNA-seq data to delineate cell-type proportion changes,

242 which could be borrowed to answer the same question. However, whenever there are ten or

243 more sub-cell types, the results from deconvolution methods become unreliable. In contrast,

244 LRcell enables comparison across many more cell types which is important for complex

245 tissues such as brain.

246 The development of LRcell is inspired by LRpath [16], which is designed for linking

247 experimental changes to biological pathways or a pre-defined gene set. In LRcell, we treat 248 cell-type specific marker genes as gene sets and calculate the enrichment of each cell type 
when comparing two biological conditions. We believe that the most enriched cell type(s) is highly likely to play an important role in the experimental condition change.

\section{scRNA-seq Data Preprocessing}

253 In this study, we include marker genes from mouse whole brain, human prefrontal cortex

254 (pFC) and human PBMC, along with 66 cell types' markers from four tissues (midbrain, cord blood, ovary and skeletal muscle) adopted from MSigDB. For each scRNA-seq dataset, we first retrieved raw read count matrix. Next, we filtered out low-quality cells and genes and apply column-wise normalization and log transformation on the data.

[17], contains nine brain regions from adult mice. The data provided has already been prefollowing the original study.

The human pFC scRNA-seq dataset [18], produced by 10X Genomics Chromium, is derived from the $\mathrm{pFC}$ region (specifically BA9). The dataset contains two conditions: healthy controls and major depressive disorder (MDD). We split the data matrix into two parts and filtered out cells expressing less than 10 genes and genes expressed in less than 10 cells respectively. We also filtered out mitochondrial, ribosomal genes, and genes from annotation clusters (Astros_1, Mix_1, Mix_2, Mix_3, Mix_4, Mix_5, Inhib_4_SST).

269 The PBMC dataset [8], generated by CITE-seq technology [19], is derived from an HIV vaccine trial study which involves eight volunteers at three timepoints: immediately before,

271 three days and seven days after the vaccine. The study contains 161,764 cells in total. To

272 accelerate the marker gene selection, we separated the count matrix according to the time 
label and filtered out low-quality cells and genes (mitochondrial, ribosomal genes and those expressed in less than 1,000 cells). The cluster annotated as "Doublet" was filtered out.

275

\section{Marker Gene Selection}

277 After obtaining the log-normalized gene expression matrix along with high-quality sub-cell type clusters, we calculated the enrichment scores for each cluster (sub-cell type) using the marker gene selection method described in Marques et al[20]. The cluster-specific gene enrichment is defined as the average gene expression levels of cells in that cluster divided by the average gene expression levels in all cells. The enrichment score is adjusted by introducing a penalty representing the fraction of cells in that cluster expressing the marker gene. Combined, this score allows the identification of genes with cluster-specific high expression values to be selected as marker genes. The description below is adapted from the original publication. $i=1, \ldots, M, j=1, \ldots, L, k=1, \ldots, N_{j}$ and $N=\sum_{j=1}^{L} N_{j}$. The overall average expression of the ith gene across all cells is defined as:

$$
\bar{E}_{i . .}=\frac{1}{N} \sum_{j=1}^{L} \sum_{k=1}^{N_{j}} E_{i j k}
$$

291 The average expression of gene $i$ in the $j$ th cluster is defined as:

$$
\bar{E}_{i j .}=\frac{1}{N_{j}} \sum_{k=1}^{N_{j}} E_{i j k}
$$

The enrichment for gene $i$ in the $j$ th cluster as:

$$
\text { Enrichment }_{i, j}=\frac{\bar{E}_{i j}}{\bar{E}_{i .}}
$$


295 Next, we consider the proportion expressing the gene $i$ in the $j$ th cluster as:

$$
\operatorname{Prop}_{i, j}=\frac{1}{N_{j}} \sum_{k=1}^{N_{j}} I\left(E_{i j k}>0\right)
$$

The $I(\cdot)$ is an indicator function.

The enrichment score for gene $i$ in the $j$ th cluster is computed as:

$$
\text { Score }_{i, j}=\text { Enrichment }_{i, j} \times\left(\text { Prop }_{i, j}\right)^{\text {power }}
$$

300 where "power" is a hyperparameter to be tuned manually to control the penalization for the

301 cell cluster proportion term. The power parameter is set to 1 throughout this study. After

302 calculating the weighed gene enrichment scores in each cluster, we ranked genes based on the

303 scores and selected the top 100 genes as the marker genes for each cluster.

\section{MSigDB Marker Genes}

306 We downloaded cell marker gene sets from MSigDB category C8 - cell type signature gene sets. Since not all tissue types are suitable for LRcell, we applied the following criteria to select tissues: 1) non-fetal tissues; 2) have more than 8 (sub-) cell types; 3 ) minimum number of marker gene greater than $50 ; 4)$ median number of marker genes greater than 80 . In the end, four tissue types, the midbrain, cord blood, ovary and skeletal muscle remain.

\section{Bulk RNA-seq Data Preprocessing}

313 The raw count of mouse bulk RNA-seq study on neurodegenerative dementia was

314 downloaded from GEO (Accession number: GSE90693). DE analysis is performed using

315 DESeq2 [21] to obtain DEGs in each brain region.

The raw count of bulk RNA-seq study on PTSD was downloaded from Recount2

317 [22]. We extracted out the experiment contrasting PTSD cases and healthy controls with

318 timepoint of pre-employment and performed DESeq2 to obtain DEGs. 


\section{LRcell Analysis}

321 LRcell is inspired by LRpath, which was designed for identifying sets of predefined gene sets

322 that show enrichment with differentially expressed transcripts in microarray experiments.

323 LRcell uses logistic regression or linear regression to assess whether marker genes (as defined in the Marker Gene Selection sub-section above) of a specific cell type are more likely to be DE genes in a particular bulk RNA-seq study. The linear regression option is added to handle the continuous enrichment scores of marker genes. Users can choose accordingly. To facilitate our analysis, we assume that the major sub cell types of the tissue their marker genes are known a priori.

Then LRcell runs a logistic regression as

$$
\log \frac{\theta}{1-\theta}=\alpha+\beta x
$$

and

$$
\theta=P(Y=1)
$$
represents the chance that the gene is a marker gene. We use $-\log (p-$ value $)$ as the explanatory variable $x$. Whether a specific cell type is involved in the experimental condition change is evaluated by testing the null hypothesis that $\beta=0$ against the alternative that $\beta \neq$ which sub cell type(s) drives the changes.

$$
Y=\alpha+\beta x
$$


where $Y$ indicates the enrichment scores of genes. Same as logistic regression, the p-value

344 can be obtained from testing the null hypothesis that $\beta=0$ against the alternative that $\beta \neq 0$

345 using the t-test.

Once the p-values are obtained, we calculate False Discover Rate (FDR) using p.adjust() function in $\mathrm{R}$ to adjust p-values with Benjamini-Hochberg method.

\section{Input and Output}

350 LRcell requires two inputs: (1) a ranked list of genes with DE p-values in a bulk RNA-seq experiment; (2) sets of marker genes from all sub-cell types of the bulk tissue acquired from scRNA-seq datasets a priori or from MSigDB C8 - cell type signature gene sets. For those cell markers derived from scRNA-seq datasets, we offer choices for users to choose between species as human or mouse and the region indicates the specific brain region or PBMC. For MSigDB cell markers, we store the marker genes into the LRcellTypeMarkers packages which can be easily downloaded. When running LRcell() function, the Logistic Regression (LR) option is set as the default, while users can also set the method option as LiR if Linear Regression is desired. For linear regression, an enrichment score is needed as input for each gene whereas gene sets are sufficient for logistic regression. For MSigDB cell type signature gene set, LR option is

360 recommended as there is no enrichment score information available. For customized input, i.e., a 361 scRNA-seq data, we offer a LRcell_gene_enriched_scores function which takes the read counts 362 matrix and cell annotation as input to generate enrichment scores for genes in each cell type. For

363 further subsetting, get_markergenes can be used for generating marker genes for more specific

364 sub cell types.

365 The output is a list of significance p-value (or FDR), one for each sub cell type. For

366 visualization, LRcell produces Manhattan plot, which can be drawn through

367 plot_manhattan_enrich function. We also provided a plot (plot_marker_dist function) indicating

368 where certain cell-type specific marker genes locate on the bulk DE genes. The bulk DE genes 
are sorted using $-\log 10(\mathrm{p}$-value $) \times \operatorname{sign}(\log 2$ FoldChange $)$ which could potentially give available at http://bioconductor.org/packages/release/bioc/html/LRcell.html.

\section{Declarations}

\section{Ethics approval and consent to participate}

Not applicable.

\section{Consent for publication}

381 Not applicable.

\section{Availability of data and materials}

384 The datasets analyzed during the current study are available in the Gene Expression Omnibus

385 (GEO) with following accession numbers: mouse whole brain [5] (GSE116470), human pFC

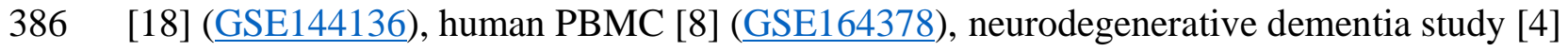

387 (GSE90696) and PTSD study [7] (GSE64814). The R package is freely available on

388 Bioconductor (https://doi.org/doi:10.18129/B9.bioc.LRcell) and the external marker genes

389 are stored in another R package named LRcellTypeMarkers on Bioconductor

390 (https://doi.org/doi:10.18129/B9.bioc.LRcellTypeMarkers). 
393 The authors declare that they have no competing interests.

394

\section{Author's contributions}

396 ZQ and WM conceived the idea and supervised the study. WM realized the method and

397 developed the R package. WM analyzed the real data and conducted the computational

398 experiments. SS provided guidance on validating biological experiments and tested the

399 package. WM and ZQ summarized the results and wrote the manuscript. All authors have

400 read and approved the manuscript. 


\section{References}

402

403

404

405

406

407

408

409

410

411

412

413

414

415

416

417

418

419

420

421

422

423

424

425

426

427

428

429

430

431

432

433

434

435

436

437

438

1. Newman AM, Steen CB, Liu CL, Gentles AJ, Chaudhuri AA, Scherer F, et al.

Determining cell type abundance and expression from bulk tissues with digital cytometry. Nat Biotechnol. 2019;37:773-82.

2. Subramanian A, Tamayo P, Mootha VK, Mukherjee S, Ebert BL, Gillette MA, et al. Gene set enrichment analysis: A knowledge-based approach for interpreting genome-wide expression profiles. PNAS. 2005;102:15545-50.

3. Liberzon A, Birger C, Thorvaldsdóttir H, Ghandi M, Mesirov JP, Tamayo P. The Molecular Signatures Database (MSigDB) hallmark gene set collection. Cell Syst. 2015;1:417-25.

4. Swarup V, Hinz FI, Rexach JE, Noguchi K, Toyoshiba H, Oda A, et al. Identification of evolutionarily conserved gene networks mediating neurodegenerative dementia. Nature medicine. Nature Publishing Group; 2019;25:152-64.

5. Saunders A, Macosko EZ, Wysoker A, Goldman M, Krienen FM, Rivera H de, et al. Molecular Diversity and Specializations among the Cells of the Adult Mouse Brain. Cell. 2018;174:1015-1030.e16.

6. Perry VH, Nicoll JA, Holmes C. Microglia in neurodegenerative disease. Nature Reviews Neurology. Nature Publishing Group; 2010;6:193.

7. Breen MS, Maihofer AX, Glatt SJ, Tylee DS, Chandler SD, Tsuang MT, et al. Gene networks specific for innate immunity define post-traumatic stress disorder. Molecular psychiatry. Nature Publishing Group; 2015;20:1538-45.

8. Hao Y, Hao S, Andersen-Nissen E, Mauck WM, Zheng S, Butler A, et al. Integrated analysis of multimodal single-cell data. Cell. 2021;184:3573-3587.e29.

9. Ong S-M, Teng K, Newell E, Chen H, Chen J, Loy T, et al. A novel, five-marker alternative to CD16-CD14 gating to identify the three human monocyte subsets. Frontiers in immunology. Frontiers; 2019;10:1761.

10. Kratofil RM, Kubes P, Deniset JF. Monocyte Conversion During Inflammation and Injury. Arterioscler Thromb Vasc Biol. 2017;37:35-42.

11. Kuan P-F, Yang X, Clouston S, Ren X, Kotov R, Waszczuk M, et al. Cell type-specific gene expression patterns associated with posttraumatic stress disorder in World Trade Center responders. Transl Psychiatry. 2019;9:1.

12. Gong T, Szustakowski JD. DeconRNASeq: a statistical framework for deconvolution of heterogeneous tissue samples based on mRNA-Seq data. Bioinformatics. 2013;29:1083-5.

13. Wang X, Park J, Susztak K, Zhang NR, Li M. Bulk tissue cell type deconvolution with multi-subject single-cell expression reference. Nat Commun. 2019;10:1-9.

14. Baron M, Veres A, Wolock SL, Faust AL, Gaujoux R, Vetere A, et al. A single-cell transcriptomic map of the human and mouse pancreas reveals inter-and intra-cell population structure. Cell systems. Elsevier; 2016;3:346-360. e4. 

composition from gene expression data. Nat Commun. 2019;10:1-9.

441 16. Sartor MA, Leikauf GD, Medvedovic M. LRpath: a logistic regression approach for identifying enriched biological groups in gene expression data. Bioinformatics. 2009;25:2117.

444 17. Macosko EZ, Basu A, Satija R, Nemesh J, Shekhar K, Goldman M, et al. Highly parallel genome-wide expression profiling of individual cells using nanoliter droplets. Cell. Elsevier; 2015;161:1202-14.

447 18. Nagy C, Maitra M, Tanti A, Suderman M, Théroux J-F, Davoli MA, et al. Single-nucleus transcriptomics of the prefrontal cortex in major depressive disorder implicates oligodendrocyte precursor cells and excitatory neurons. Nature neuroscience. Nature

450 Publishing Group; 2020;23:771-81.

451 19. Stoeckius M, Hafemeister C, Stephenson W, Houck-Loomis B, Chattopadhyay PK, 452 Swerdlow $\mathrm{H}$, et al. Simultaneous epitope and transcriptome measurement in single cells. 453 Nature Methods. 2017;14:865-8.

454 20. Marques S, Zeisel A, Codeluppi S, van Bruggen D, Falcão AM, Xiao L, et al. Oligodendrocyte heterogeneity in the mouse juvenile and adult central nervous system. Science. American Association for the Advancement of Science; 2016;352:1326-9.

457 21. Love MI, Huber W, Anders S. Moderated estimation of fold change and dispersion for 458 RNA-seq data with DESeq2. Genome Biol. 2014;15:550.

459 22. Collado-Torres L, Nellore A, Kammers K, Ellis SE, Taub MA, Hansen KD, et al. 460 Reproducible RNA-seq analysis using recount2. Nat Biotechnol. 2017;35:319-21. 
Figure 1 Overview of $\mathbf{L R}$ cell workflow. (A) Bulk RNA-seq experiment design with control and disease conditions. (B) Illustration of a marker gene list derived from scRNA-seq experiment. (C) (upper panel) Illustration of how the distribution of marker genes on bulk DE genes may affect the regression; (lower panel) Illustration of the FDR rate for each cell type in which the grey cells and dark blue cells are most likely involved in the condition change.

Figure 2 LRcell datasets and applying LRcell to real cases. (A) summary of the all tissue types in which marker genes have been pre-embedded in LRcell. (B) LRcell result of mapping the bulk neurodegenerative dementia DE genes to the mouse brain frontal cortext (FC) region . Microglia is shown as the most significant cell type. (C) GSEA result of mapping the bulk neurodegenerative dementia DE genes using the same marker genes for LRcell (mouse brain FC) as input. We see numerous dots have the same significance level which is difficult to interpret. (D) Cell type proportions for control and disease samples calculated by MuSiC. Each box contains 17 individuals. The $\mathrm{x}$-axis is ordered by the t-test significance between the two conditions. (E) LRcell result of mapping bulk PTSD DE genes to human PBMC. CD16+ Monocytes is shown as the most significant cell type. (F) GSEA result of mapping bulk PTSD DE genes to human PBMCusing the same marker genes for LRcell (human PBMC) as input. Again, we see numerous dots have the same significances. (G) Cell type proportions for control and disease samples calculated by MuSiC. The $\mathrm{x}$-axis is ordered by the t-test significance between two conditions. As shown in the figures, MuSiC failed to identify all cell type proportions as there are plenty of 0 s starting from ASCD_pDC cell type and the CD14+ Monocytes are wrongly inferred.

Figure S1: Marker genes overlap between different brain regions. Top 100 marker genes are selected for each cell type, thus, the maximum overlap in these figures is 100. (A) heatmap illustrating the overlap of marker genes among cell types within the Frontal Cortex (FC) region derived from mouse whole brain scRNA-seq dataset. The highlighted area describes the overlap between FC_11-3.unknown, FC_11-4.unknwon and FC_11-1.Microglia as an illustration for the similarity between these three sub-cell types. (B) Heatmap illustrating the overlap of marker genes among cell types within the FC and cell types within the Cerebellum (CB). (C) Heatmap illustrating the overlap of marker genes among cell types within the FC and cell types within the Hippocampus (HC). As shown in (B) and (C), the overlaps between cell types belong to different brain regions are lower than overlaps between cell types belong to the same brain region.

Figure S2: Marker genes overlap across time points from PBMC dataset. Top 100 marker genes are selected for each cell type and the maximum overlap in figures is 100. (A) Heatmap illustrating the overlap of the cell-type-specific marker genes between time point 0 and time point 3. (B) Heatmap illustrating the overlap of the cell-type-specific marker genes between time point 3 and time point 7 . As shown in the figure, the diagonal pattern indicates the marker genes selected are highly cell-type-specific and shared across time points.

\section{Figure S3. Comparison of LRcell analysis results when the tissue from which the DE} gene lists is obtained match or un-match the tissue type from where the marker genes are collected. LRcell result when: (A) Both DE gene list and marker genes are obtained from HC. (B) Both DE gene list and marker genes are obtained from CB. (C) DE gene list obtained from $\mathrm{HC}$ and marker genes collected from FC. (D) DE gene list obtained from CB and 
521 marker genes collected from FC. (E) DE gene list obtained from CB and marker genes 522 collected from HC. (F) DE gene list obtained from FC and marker genes collected from HC. 523 (G) DE gene list obtained from FC and marker genes collected from CB. (H) DE gene list obtained from HC and marker genes collected from CB. Compared to (D) and (E), which analyze CB bulk DE genes, (B) has a higher resolution as the FDR ranges from 0 to 12. 
A

Control
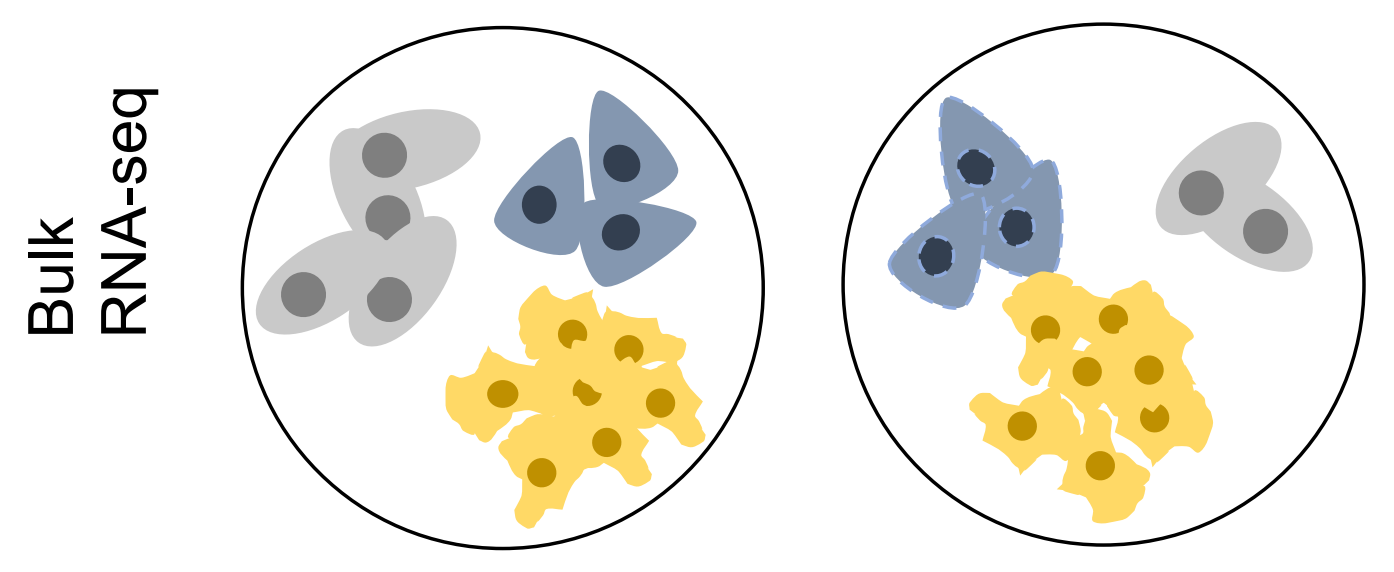

B

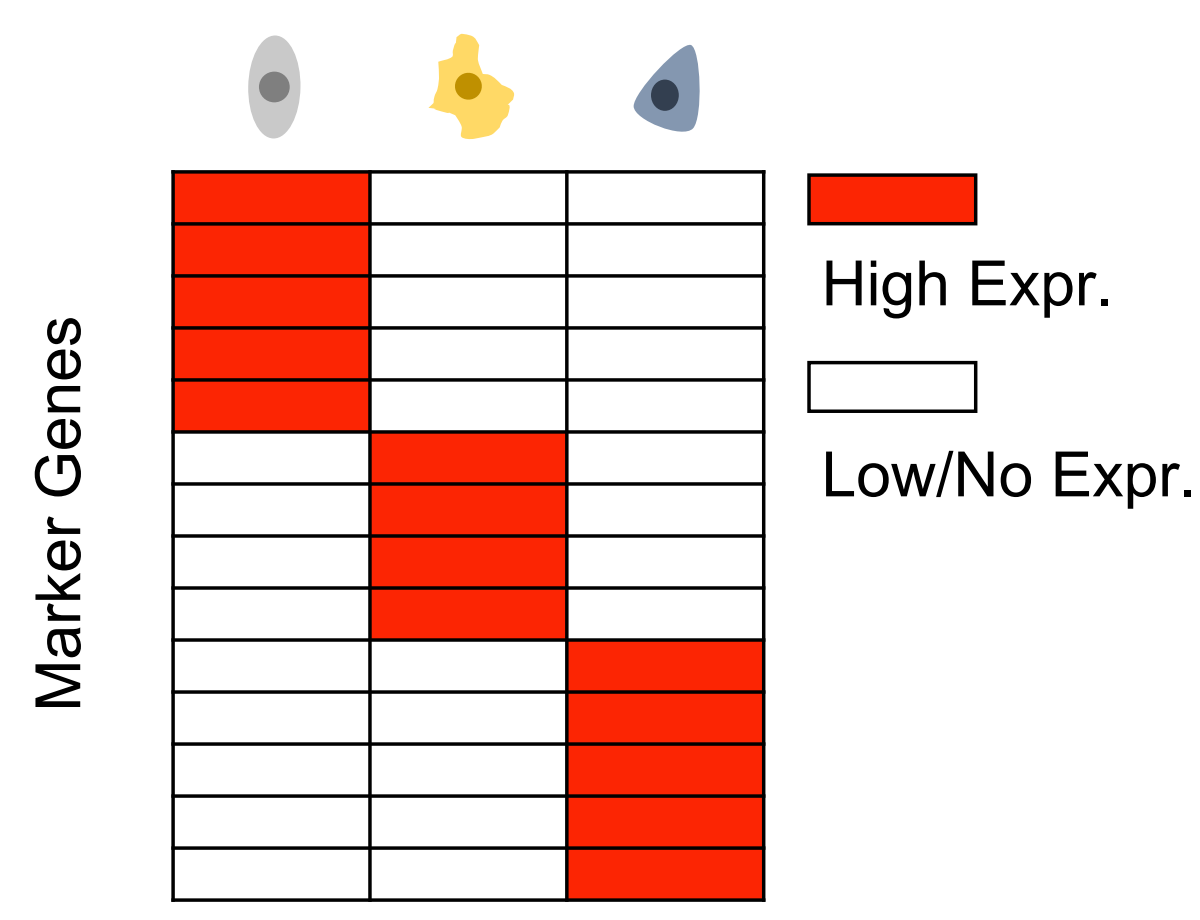

C
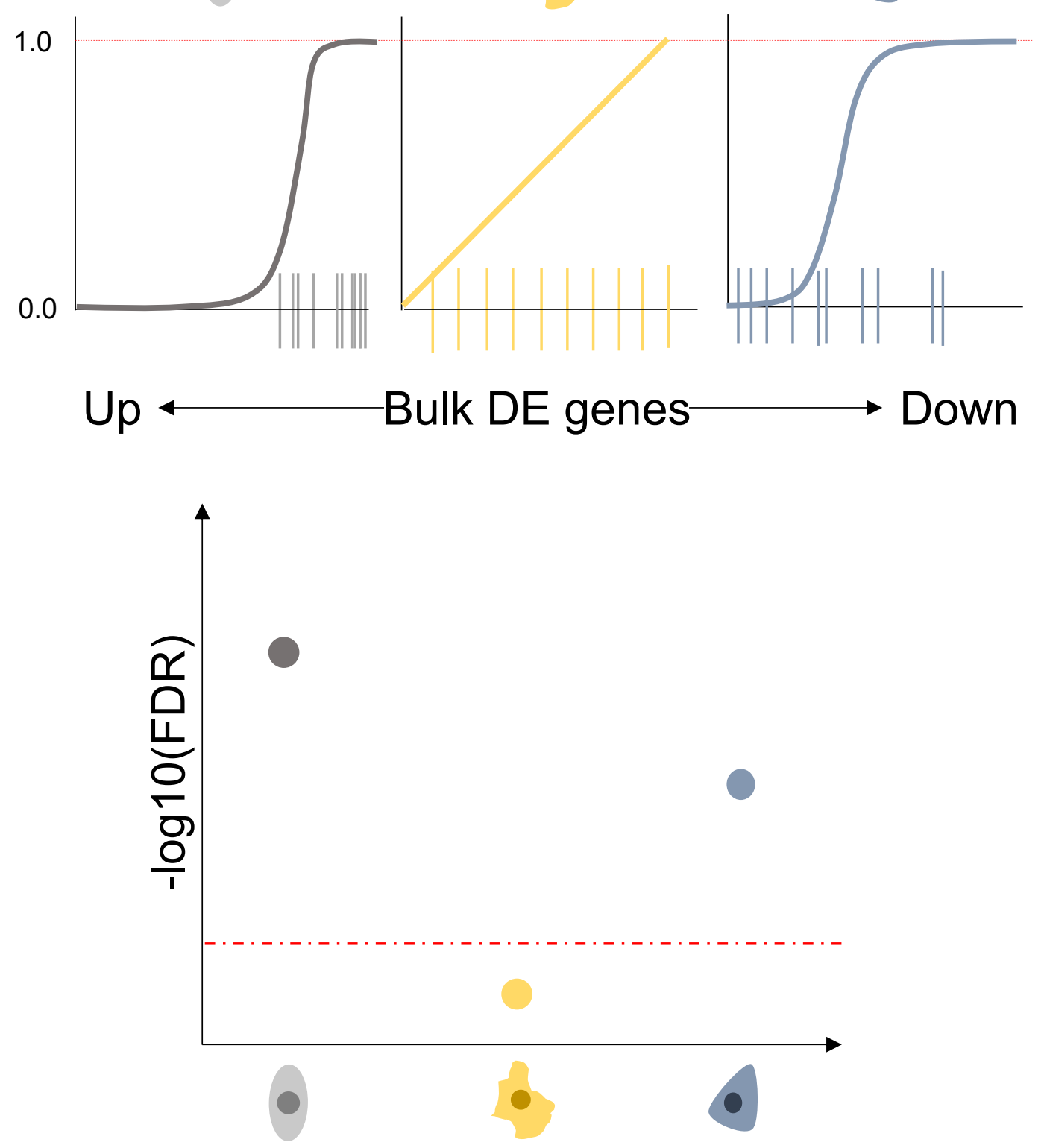

LRcell result 
A

Human $T^{\text {Blood }} T^{\text {PBMC }}$

L Cord Blood (MSigDB)

Brain $[$ pre-Frontal Cortex - BA8/9 region

Ovary (MSigDB)

Skeletal Muscle (MSigDB)

Mouse - Brain - Frontal Cortex

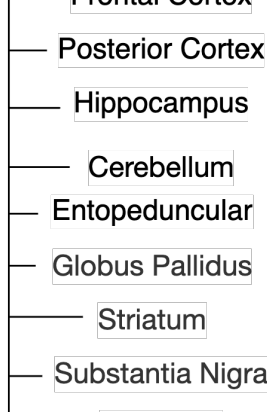

Substantia Nigra

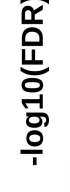

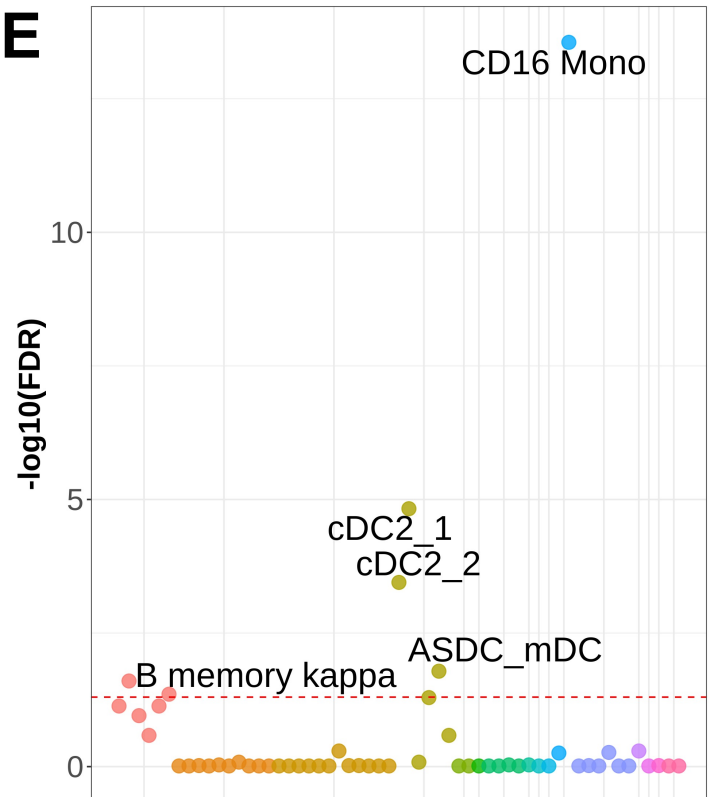

clusters
D
Astrocytes
Endothelial
FibroblastLike

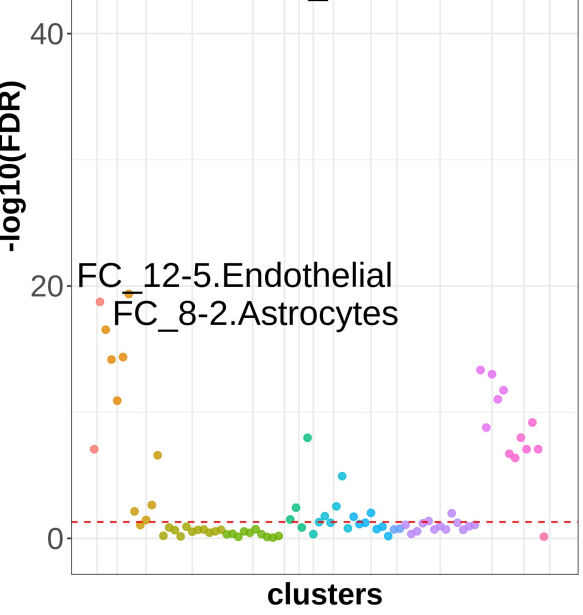

Interneuron_CGE
Interneuron_MGE
Microglia

Mural
Neuron_Claustrum
Neuron-L2/3

Neuron- L5
Neuron-L5b

Olirodendrocytes
Polydendrocytes

Polydendrocytes
unknown
Mouse Brain FC Cell Types
Mouse Brain FC Cell Types

Astrocytes
Endothelial
Fibroblastlike

ElbroblastLike
Interneuron-CGE

Mural _Claustrum

Neuron-25

Neuron L6
Oligodendrocytes
Polydendrocytes

Polydendrocytes
unknown

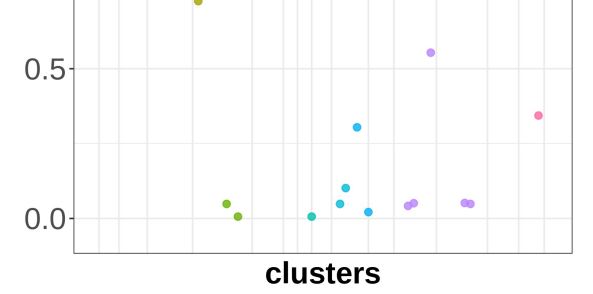

unkown

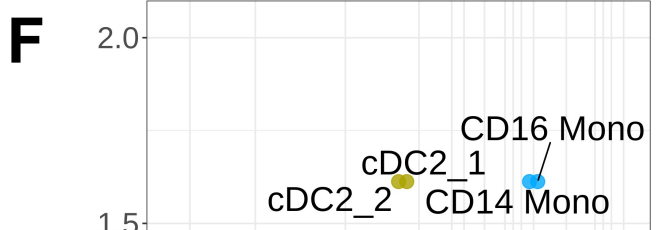

- B cells

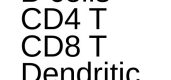

Dendritic
Double Negative T

Double Negative
Eryth
Gamma Delta T

HSPC

MAIT
Monocytes
NK cells
Plasma

Plasma
Plasmablast

Plasmablast
Platelet
Regulatory T
PBMC time0 marker genes

- B cells

Dendritic
Double Negative T

Gamma Delta T

ILC
MAIT

MAIT
Monocytes
NK cells

Plasmablast

Plasmablast
Platelet
Regulatory T
Interneuron_MG
Microglia
Mural

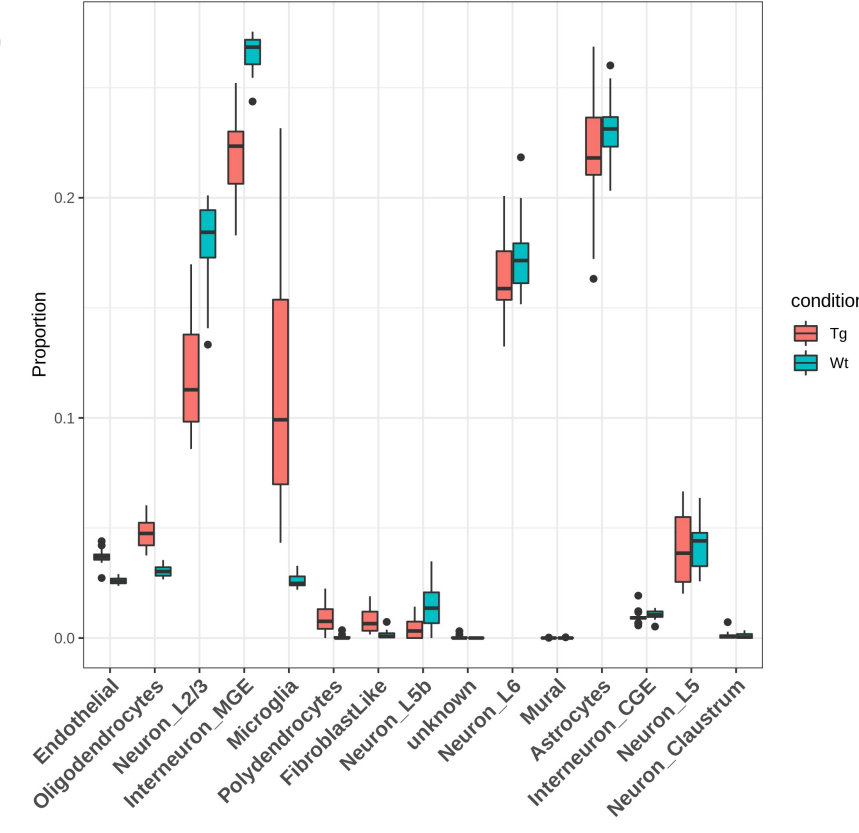

G

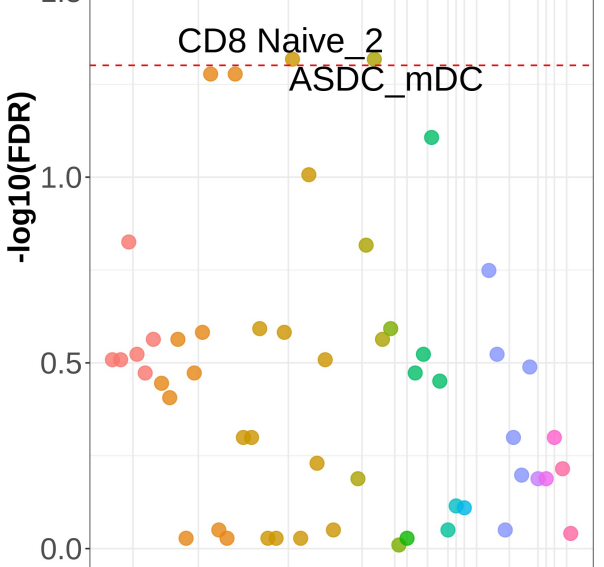

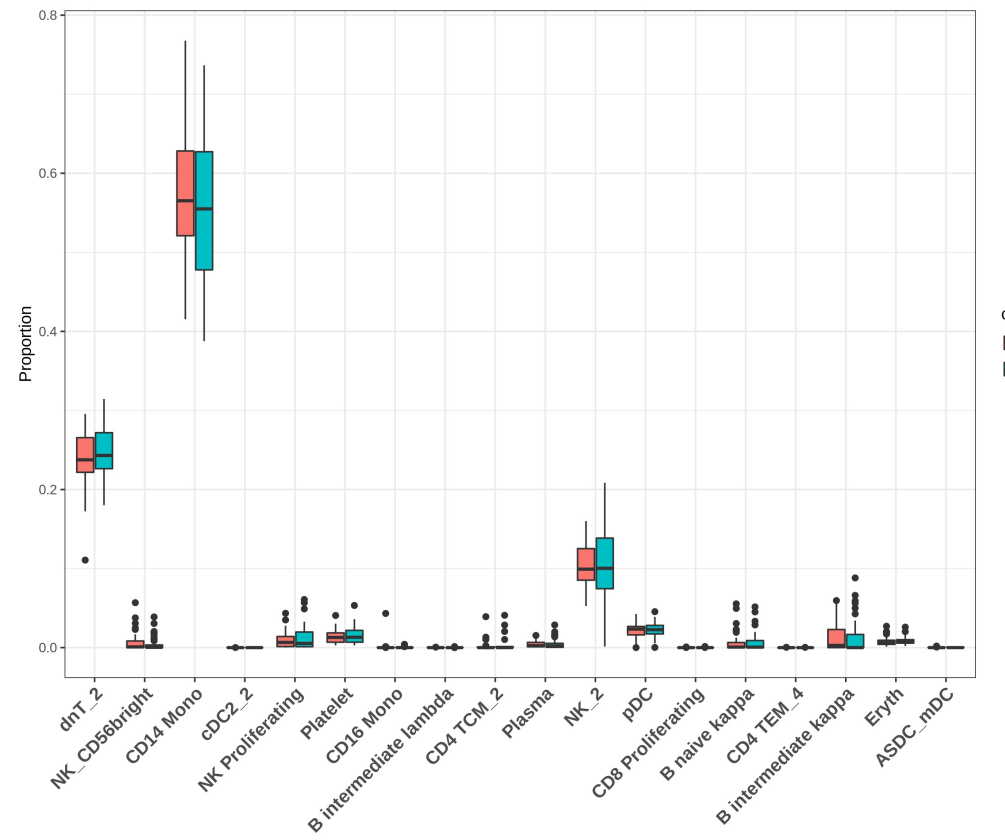




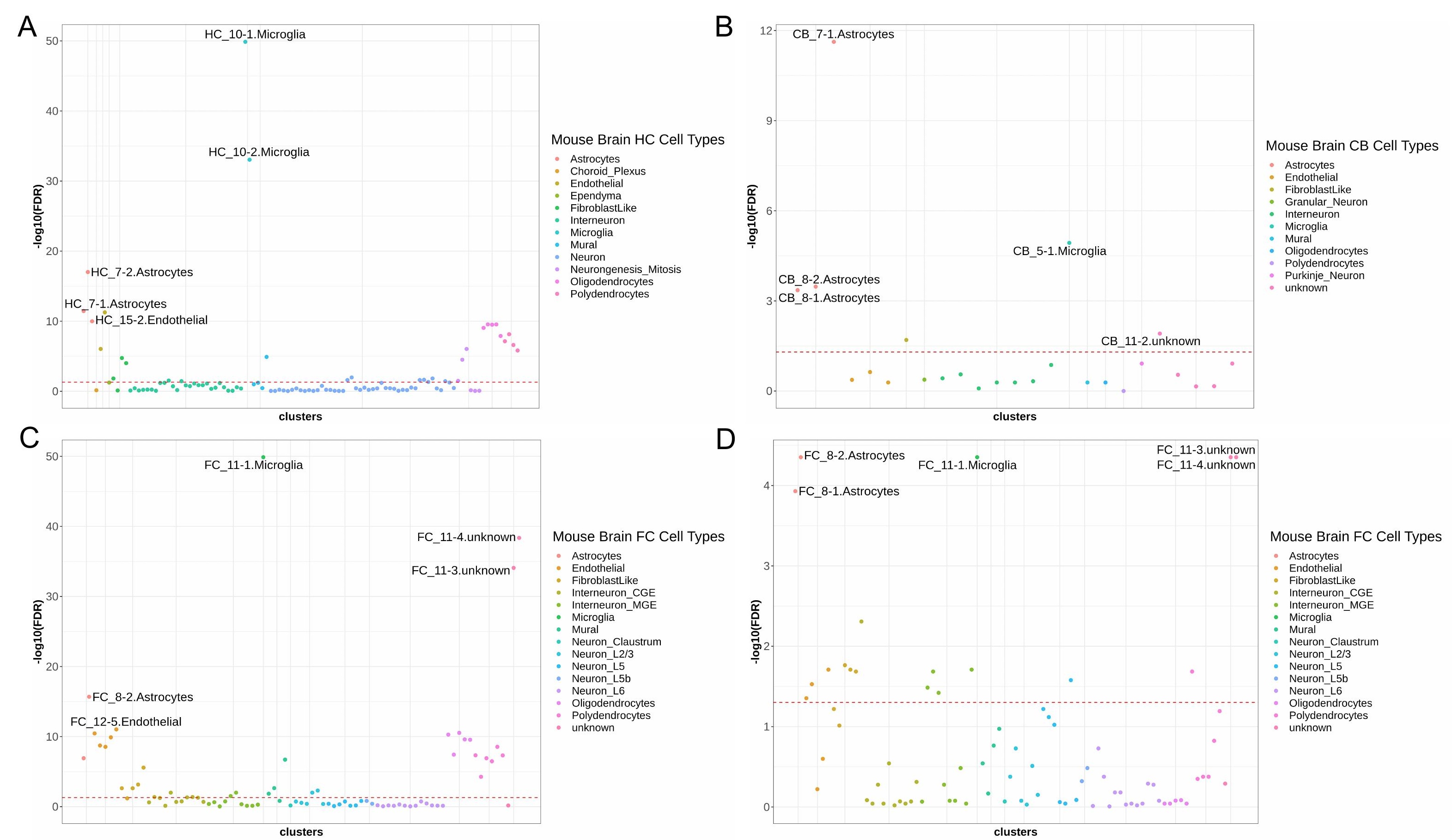




\section{E}

1.Astrocytes ${ }^{\circ} \mathrm{HC}$ 10-1.Microglia HC_7-3.Astrocytes ${ }^{2}$ HC_10-2.Microglia

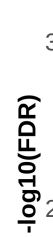

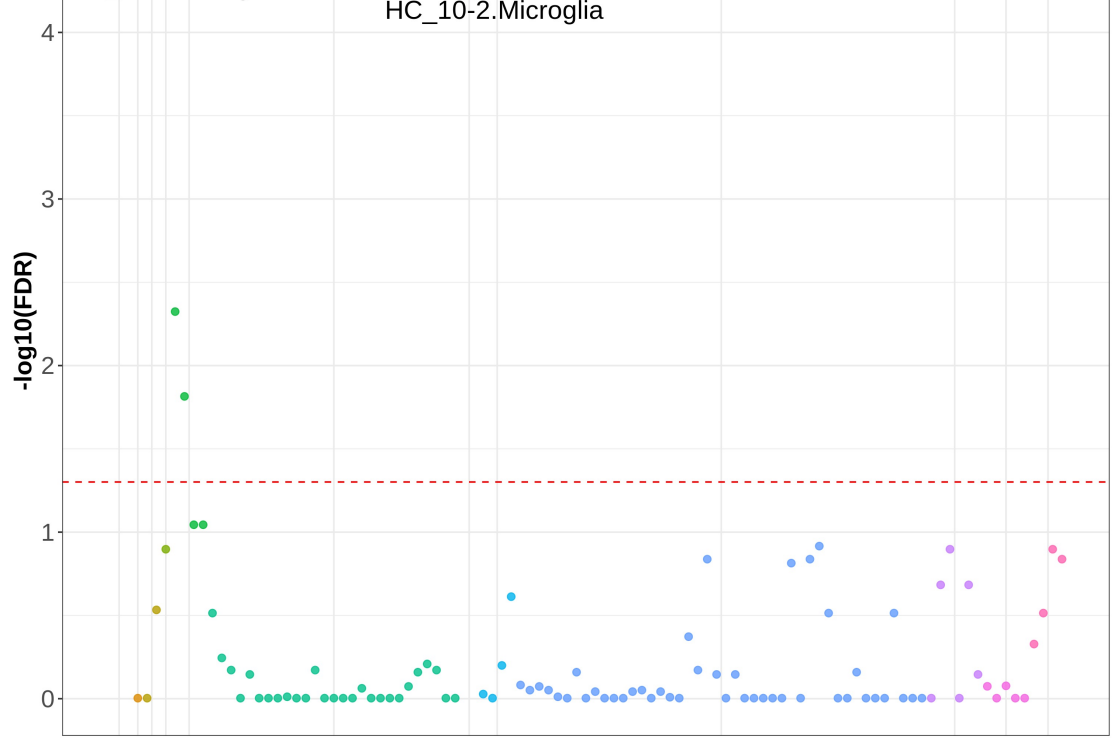

$\mathrm{G}^{60}$

CB_7-1.Astrocytes

. . CB_10-3.Endothelia

clusters

B_5-1.Microglia

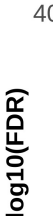

CB 6-1.Oligodendrocytes
Mouse Brain HC Cell Types

Astrocytes
Choroid Plex

- Endothelial

Ependyma

FibroblastLik

Interneuron

Mural

Neuron

Neurongenesis_Mitosis

Oligodendrocytes

Mouse Brain CB Cell Types

- Astrocytes

- FibroblastLike

Granular_Neuron

- Microglia

- Oligodendrocytes
- Polydendrocytes
- Purkinje_Neuron

- Purkinje_N

F

HC_10-2.Microglia

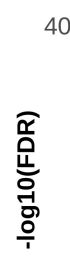

20- HC_15-2.Endothelial HC_7-2.Astrocytes

-

-
HC 8-2.Oligodendrocytes HC_8-4.Oligodendrocytes

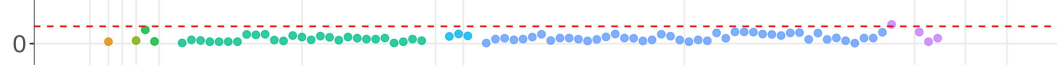

$\mathrm{H}$

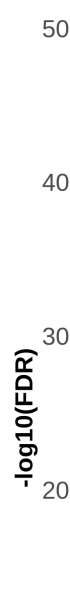

10-CB_8-2.Astrocytes

CB_8-1.Astrocytes
Mouse Brain HC Cell Types

- Astrocytes

Choroid_Plexus
Endothelial

Endothelial
Ependyma

Fibroblastlike

Interneuron
Microglia

Mural

Neuron

Neurongenesis_Mitosis

Oligodendrocytes
Mouse Brain CB Cell Types

- Astrocytes

Fibroblastlike

Granular_Neuron

Interneuron

Mural

Oligodendrocytes

- Polydendrocytes

- Purkinje_Neuron 